\title{
Continuous and Scalable Fabrication of Transparent Conducting Carbon Nanotube Films
}

\author{
Budhadipta Dan, ${ }^{\dagger}$ Glen C. Irvin, ${ }^{\neq}$and Matteo Pasquali ${ }^{\dagger, *}$ \\ ${ }^{\dagger}$ Department of Physics and Astronomy, Department of Chemical and Biomolecular Engineering, and Department of Chemistry, The Smalley Institute for Nanoscale \\ Science \& Technology, Rice University, 6100 Main Street, Houston, Texas 77005, and ¥Unidym Inc., 1430 0’Brien Drive, Suite G, Menlo Park, California 94025
}

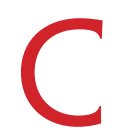
arbon nanotubes (CNTs) combine nanoscale size with high aspect ratio $(\sim 1000)$ and unique electrical, optical, mechanical, and electrochemical properties, ${ }^{1-6}$ making them ideal candidate materials for high-impact applications in various fields. ${ }^{7}$ Yet, much as in polymer science and engineering, such applications can only be attained by developing appropriate scalable processes that translate the properties of the elemental molecules (SWNTs) or particles (CNTs) into macroscopic materials. Scientific knowledge about processing SWNTs into macroscopic and commercially useful products is still scarce and is a topic of extensive current research. An important recent success in this area has been the fabrication of optically transparent and electrically conducting thin films of pure SWNTs. Such transparent and conductive thin films or coatings may replace indium tin oxide (ITO) in a wide range of applications, for example, in touch screens, flat panel displays, image recorders, optical communication devices, and solar cells. ${ }^{8,9}$

Nanotube films have been fabricated by vacuum filtration, ${ }^{10-12}$ transfer printing onto various substrates, ${ }^{13,14}$ drawing from vertically oriented nanotube forests, ${ }^{15}$ spin coating ${ }_{1}^{16}$ drop casting from SWNT dispersions, ${ }^{17}$ quasi-Langmuir-Blodgett deposition, ${ }^{18}$ dip-coating, ${ }^{19}$ direct CVD growth, ${ }^{20}$ air-spraying, ${ }^{21,22}$ and, after suitable functionalization, wire-wound rod coating, ${ }^{23,24}$ and slot coating. ${ }^{25}$ However, most of the processes proposed so far cannot be ported easily to large scale production-with the exception of air-spraying, which has the drawback of forming sparse and relatively nonuniform networks, ${ }^{22}$ and rod and slot coating, which are scalable methods but have so far have required functionalized

\begin{abstract}
We report an industrially scalable, fast, and simple process for the large scale fabrication of optically transparent and electrically conducting thin films of single-walled carbon nanotubes (SWNT). Purified, pristine HiPco SWNTs were dispersed in water at high concentrations with the help of surfactants, rod-coated into uniform thin films, and doped by various acids. We show how to combine different surfactants to make uniform dispersions with high concentration of SWNTs and optimal rheological behavior for coating and drying, including preventing dewetting and film rupture that has plagued earlier attempts. Doping by fuming sulfuric acid yielded the films with best performance (sheet resistance of 100 and $300 \Omega / s q$ for respective transparency of $70 \%$ and $90 \%$ ). We use a figure of merit (FOM) plot for an immediate evaluation and comparison of the performance and microstructure of CNT films produced by different methods. Further scientific engineering will pave the way to the deployment of CNT films in commercial applications.
\end{abstract}

KEYWORDS: single walled carbon nanotubes $\cdot$ SWNT

films - transparent - conductive - coatings - wire-wound rod coating - rheology.

CNTs. ${ }^{23-26}$ Here we report the fabrication of films of SWNT films by "draw-down Mayer rod coating" (rod coating) process using pristine SWNTs.

Draw-down rod coating is a well-known coating technique widely used by laboratories in the coating industry for making liquid thin films in a continuous and controlled manner. ${ }^{27}$ Fluids that can be coated effectively by the Mayer rod method can then be readily adapted to more controllable, higher throughput methods such as slot, slide, and roll coating. ${ }^{28,29}$ Figure 1 a shows a schematic diagram of the rod coater with the wire-wound Mayer rod. The coating apparatus consists of a stainless steel rod wound tightly with stainless steel wire and a smooth and flat glass pad. The substrate is held down on the drawdown glass pad using heavy duty clips; the Mayer rod rolls over the substrate, doctoring off the coating fluid. Part of the liquid flows through the grooves in the wire-wound rod and forms the thin liquid film. The diameter of the wound wire determines the size of the grooves and, hence, it controls the final
*Address correspondence to
mp@rice.edu.

Received for review December 3, 2008 and accepted March 26, 2009.

Published online April 8, 2009. $10.1021 / \mathrm{nn} 8008307$ CCC: $\$ 40.75$ 


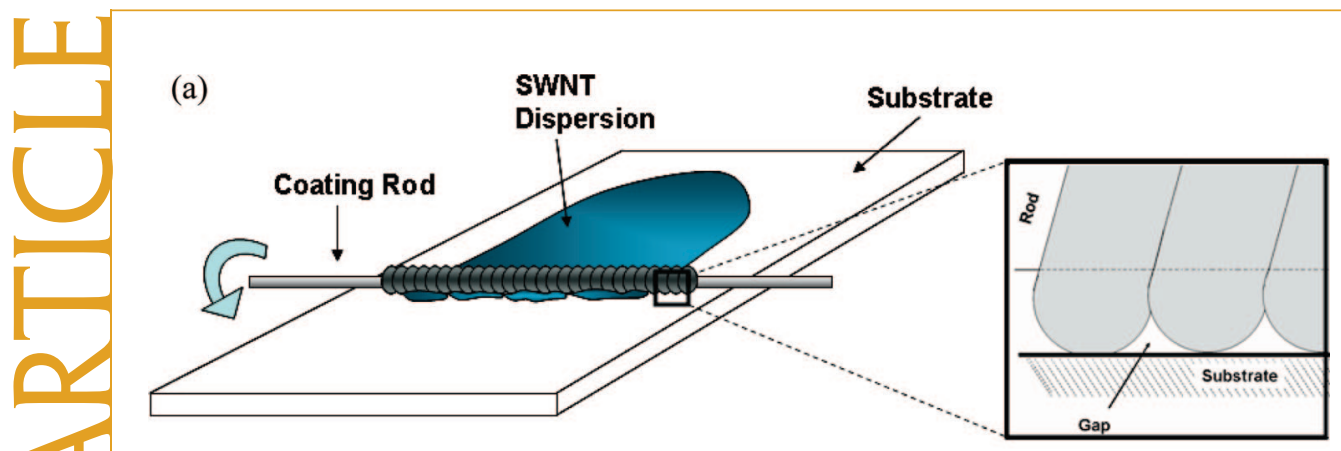

(b)

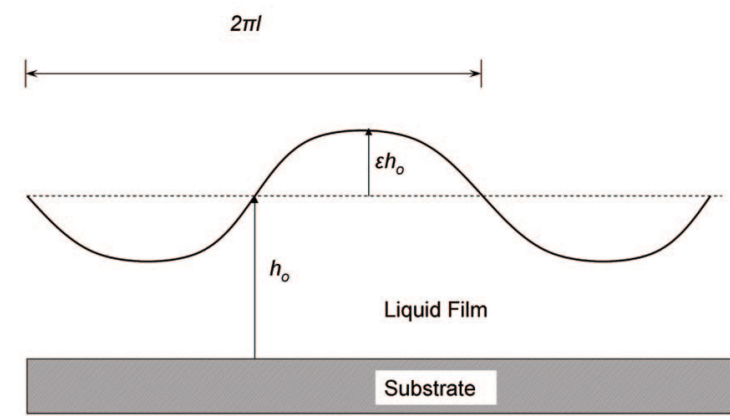

Figure 1. (a) Schematic illustration of a draw-down rod coater with a wire-wound Mayer coating rod, (b) the free surface profile of a thin film immediately after Mayer rod coating. the desired flat equilibrium state (oscillations) whereas viscous forces tend to slow down the leveling process by distributing the liquid momentum through the entire film thickness. A low Reynolds number ensures that viscous forces dominate inertial forces and any oscillations dampen out to give a flat film surface. A low capillary number ensures that capillary forces dominate the viscous forces and leveling occurs before the film can dry; equivalently, the leveling time scale $t_{\text {level }}=3 \mu /{ }^{4} /\left(\sigma h_{\circ}^{3}\right)^{30}$ is shorter than the film drying time $t_{\text {dry }}=\Delta h / J_{0}$, where $J_{0}$ is solvent evaporation current $(\mathrm{cm} / \mathrm{s})$ and $\Delta h$ is the thickness of liquid layer that must evaporate to cause gelation or solidification in the coated film. thickness of the coated liquid film. ${ }^{28}$ This technique can be used to coat directly onto polyethylene terephthalate (PET), glass, and other substrates at room temperature and in a scalable way.

A critical aspect in the rod coating process is preparation of the coating fluid, which should have specific rheological (flow) behavior and wetting properties. The coating fluid should carry sufficient solids to form a uniform and continuous layer upon drying of the coated film. The surface tension of the coating fluid should be sufficiently low to facilitate its spreading on a wide range of substrates and avoid defects like contact line recession and dewetting. After deposition, the viscosity of the coating fluid should be sufficiently high to slow secondary flows induced by surface tension and dewetting forces, allowing the film to dry without flow. The role of surface tension and viscosity in the context of coating flow can be understood by considering the capillary number $\mathrm{C} a \equiv \mu \mathrm{v} / \sigma$ and Reynolds number $R e \equiv$ $\rho v l / \mu$, where $\mu$ is the viscosity of the fluid, $v$ is the characteristic velocity, $\sigma$ is the surface tension, $\rho$ is the fluid density, and $2 \pi /$ is the wavelength of a sinusoidal disturbance.

In rod coating, the surface of the coated film is wavy, and the waves must flatten before drying (Figure 1b). The leveling process can be driven by two possible mechanisms: capillarity and gravity. For thin films (average film thickness $h_{\circ} \ll$ capillary length $\kappa^{-1} \approx(\sigma / \rho g)^{1 /}$ 2), gravitational effects are negligible compared to capillary effects. Capillary leveling is driven by a hydrostatic pressure difference, caused by surface tension forces, between the convex and concave regions of the wavy liquid. Once the liquid is set in motion, inertial forces tend to maintain the motion causing overshoot from
During the drying process, that is, the time between leveling and solidification, the liquid thin film is exposed to various stresses including those due to dewetting and nonuniform solvent evaporation; these stresses can induce secondary flows leading to contact line recession, film thinning, and rupture. A high fluid viscosity is necessary to retard the secondary flows and ensure that the film is dry before these stresses can cause any significant damage. Further details of the dewetting process are discussed in a later section. For a hand drawn rod coating process, like the one used in our case, optimal conditions require surface tension lower than $35-40 \mathrm{mN} / \mathrm{m}$ and viscosity in the range of 0.01-1 Pa.s - equivalently, $\mathrm{Ca}<0.5$ and $R e<1$, assuming a typical coating speed of $2 \mathrm{~cm} / \mathrm{s}$ and characteristic wavelength of $2 \mathrm{~mm}$ (diameter of wire on Mayer rod). ${ }^{28}$

Coating fluids are often non-Newtonian; that is, their viscosity depends on shear rate..$^{29,31,32}$ This is certainly the case for carbon nanotube dispersions, which show shear-thinning even at dilute concentrations. ${ }^{33-36}$ Coating fluids experience widely different characteristic stresses (and therefore shear rates) in different regions of the flow. In the film formation region, the liquid flows at high shear rate (normally in the shearthinning regime) through the grooves in the rod. In the coated film region, the liquid is subjected to much weaker dewetting forces; therefore, it resists flow according to its zero-shear (or low-shear) viscosity. For our hand drawn rod coating process (shown in the inset of Figure 1), the characteristic shear rate in the film formation region is $\sim 40 \mathrm{~s}^{-1}$. Therefore, the optimal coating fluid should have a viscosity in the range of $0.01-1 \mathrm{~Pa} \cdot \mathrm{s}$ at a shear rate $\leq 40 \mathrm{~s}^{-1}$. 


\section{RESULTS AND DISCUSSION}

Our coating fluids consisted of aqueous dispersions of SWNTs stabilized by surfactants. Many surfactants disperse carbon nanotubes in water; ${ }^{37}$ they differ in the stabilization mechanism (ionic vs steric, micellization vs polymeric wrapping), the maximum quantity of suspended SWNTs, the ratio of individual vs bundled SWNTs present in the dispersion, the typical size of the bundles, and their propensity for forming lightly (or strongly) flocculated structures of SWNTs and SWNT bundles. All these factors can affect the coating process as well as the structure and performance of the final film. Four surfactants were chosen on the basis of their proven effectiveness as SWNT dispersants in water: ${ }^{37}$ F108 and F98 (pluronics, polyethylene oxide, and polypropylene oxide based triblock polymers, BASF), Ssodium dodecyl sulfate (SDS, Aldrich) and sodium dodecylbenzenesulfonate (SDBS, Aldrich).

To identify the optimal surfactant, coating fluids were prepared using the above surfactants. The coating fluids were prepared by mixing $0.3-0.4 \mathrm{wt} \%$ SWNT (HiPco Rice University; HPR 166.12) with 1 wt \% of surfactant, using the technique developed by $\mathrm{O}^{\prime}$ Connell et $a^{38}{ }^{38}$ The SWNT and surfactant concentrations were chosen based on the work by Vigolo et al. ${ }^{39}$ These SWNT dispersions, as prepared, were unsuitable for coating uniform large area thin films. However, high SWNT loading (relatively higher viscosities) and thicker coated layers (20-50\% transparency) allowed us to fabricate "test" films using rod coating. The "test" films were still partially defective (due to dewetting and film rupture), but had large enough uniform regions $\left(\sim 1 \mathrm{~cm}^{2}\right)$ to allow measurement of their electrical and optical properties. The coated films were air-dried, washed vigorously to remove surfactant (see Supporting Information) and again dried overnight at $60^{\circ} \mathrm{C}$ under vacuum. Following this, all measurements were performed with the film exposed to ambient conditions.

The performance of transparent conductive thin films is typically reported as plots of sheet resistance $R_{\mathrm{s}}$ vs transparency $T$. These plots show the trade off between the transparency and conductivity for thin films using a specific material. Another intuitive way of comparing and evaluating the properties of various transparent conductive films is by using a figure of merit (FOM). The most commonly used FOMs for transparent conducting films were defined by Fraser et $a l^{40}$ and Haacke et al. ${ }^{41}$ in the form of $F_{\mathrm{tc}}=T / R_{\mathrm{s}}$ and $\Phi_{\mathrm{tc}}=T^{\mathrm{x}} / R_{\mathrm{s}}$, respectively, where $T$ is the transmittance, $R_{\mathrm{s}}$ is the sheet resistance of the thin film, and $x$ is an arbitrary integer ( $\sim 10)$ used for fixing the thickness at which maxima of $\Phi_{\mathrm{tc}}$ occurs. Both of these FOMs are functions of film thickness and are useful either for finding the optimum thickness of a transparent conductive film (for a given material) or for comparing different transparent conductive materials at a fixed film thickness.
For our analysis, we define a new FOM that is independent of thickness (for ideal films and for thickness smaller than wavelength of light) and allows immediate comparison between transparent conductors fabricated from different materials or by using different methods over the entire range of transparencies. A similar FOM was independently introduced in the recent patent literature. ${ }^{42}$ According to the Beer-Lambert law, the transmission of light $(T)$ through a film of homogeneous material can be modeled as $T=I_{1} / I_{0}=\mathrm{e}^{-\alpha t}$, where $I_{0}$ and $I_{1}$ are the intensity of incident and transmitted light, $\alpha$ is the effective absorption coefficient, and $t$ is the film thickness. The sheet resistance is defined as $R_{\mathrm{s}}=\rho / t$, where $\rho$ is the material resistivity. Combining these two equations yields

$$
R_{\mathrm{s}}=-\frac{\alpha \rho}{\ln T} \quad \text { or } \quad T=\exp \left(-\frac{\alpha \rho}{R_{\mathrm{s}}}\right)
$$

We define $(\alpha \rho)$ as the figure of merit $\left(\mathrm{FOM}_{1}\right)$. The absorption coefficient $(\alpha)$ is not a fundamental material property, but it is very "commonly used because it can be easily measured and intuitively understood". ${ }^{43}$ For a uniform and homogeneous material, $(\alpha \rho)$ is a constant parameter; therefore, sheet resistance depends linearly on $(-1 / \mathrm{In} T)$ with $\mathrm{FOM}_{1}$ as the slope (Intuitively, $\mathrm{FOM}_{1}$ is also the sheet resistance of a film with a transparency of $\mathrm{e}^{-1}=36.8 \%$ ). $\mathrm{FOM}_{1}$ is useful for comparing transparent conductive films made from different materials or by using different methods - of course, films with lower FOM have better performance.

In the special case of a metallic thin film (film thickness much smaller than wavelength of light) in air, the electro-optical properties can be modeled as ${ }^{13,43,44}$

$$
T=\left(1+\frac{2 \pi}{c} \sigma_{\mathrm{ac}} t\right)^{-2}=\left(1+\frac{Z_{0}}{2 R_{\mathrm{s}}} \frac{\sigma_{\mathrm{ac}}}{\sigma_{\mathrm{dc}}}\right)^{-2}
$$

where $\sigma_{\mathrm{dc}}$ is the DC conductivity, $\sigma_{\mathrm{ac}}$ is the optical conductivity, $c$ is the speed of light, and $Z_{0}$ is the characteristic impedance of vacuum $(\sim 376.73 \Omega)$; both $\sigma_{\mathrm{dc}}$ and $\sigma_{\mathrm{ac}}$ are fundamental properties of a material. A high value of $\sigma_{\mathrm{dc}} / \sigma_{\mathrm{ac}}$ signifies a material with high conductivity and low optical absorption and hence a better candidate for transparent conductive thin films. On the basis of the above model, the ratio $\sigma_{\mathrm{ac}} / \sigma_{\mathrm{dc}}$ can also be treated as a figure of merit $\left(\mathrm{FOM}_{2}\right) .{ }^{44}$ Performing a Taylor expansion of transparency versus sheet resistance shows that eqs 1 and 2 coincide up to first order in sheet resistance by setting $\alpha \rho=\left(Z_{0} / 2\right)\left(\sigma_{\mathrm{ac}} / \sigma_{\mathrm{dc}}\right)$ (they differ slightly at second order); they are also phenomenologically equivalent, as shown below.

Table 1 lists the FOM for our test films. Although all the films are essentially composed by SWNTs alone (with minimal residual traces of surfactants), they show substantially different electro-optical properties. In par- 


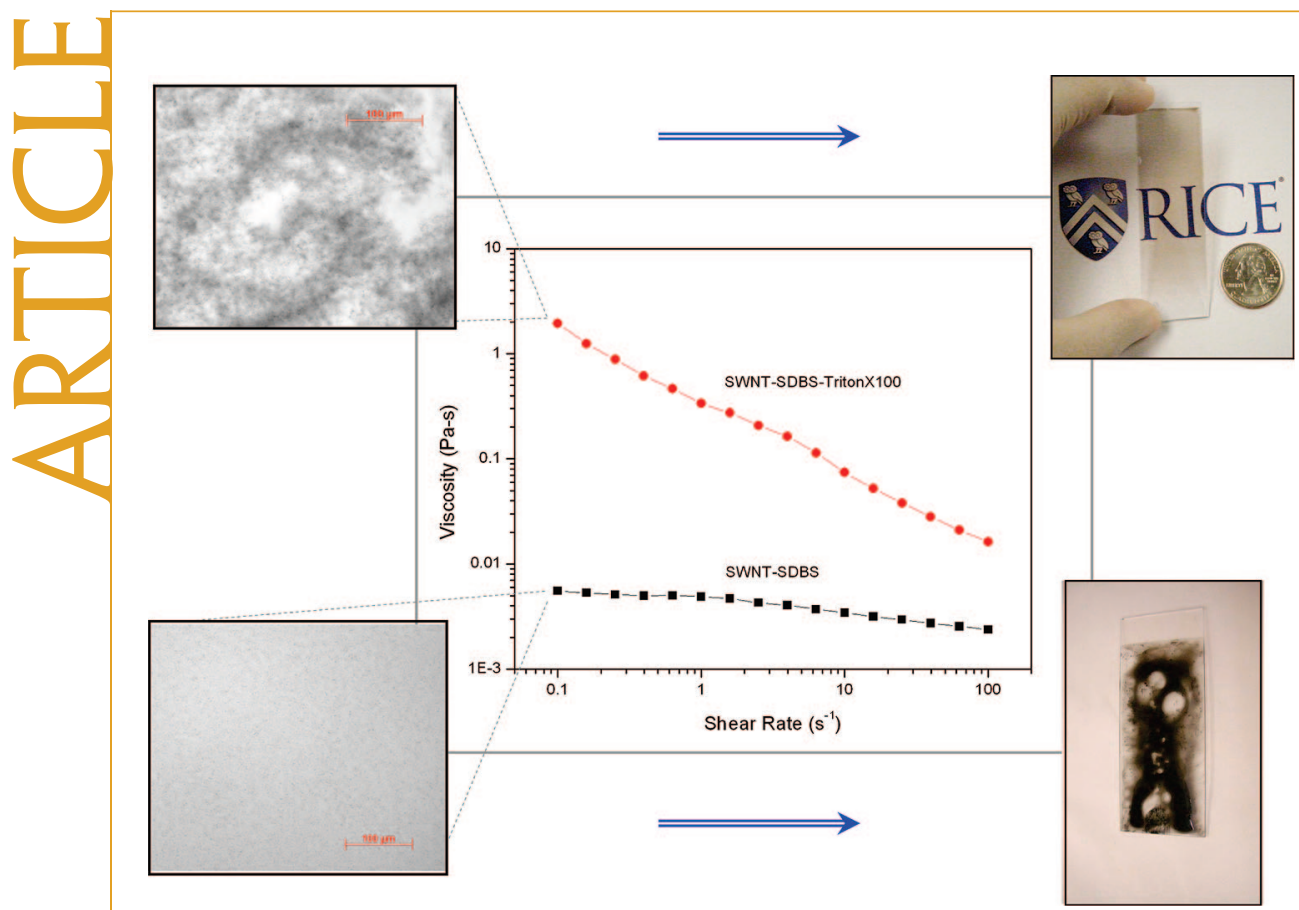

Figure 2. Viscosity vs shear rate for SWNT-SDBS dispersion and SWNT-SDBS-TX100 dispersion. The optical micrographs (left) show the different morphology of these two dispersions: in SDBS alone, the SWNTs are homogeneously dispersed as individuals and thin bundles; when TX100 is added, the SWNT and bundles form loose flocs. The formation of this network of flocs raises the low-shear viscosity by $\sim 3$ orders of magnitude. Images of the final, dry films (right) show that films formed from the SWNT-SDBS dispersion dewet and rupture, whereas films coated from the SWNT-SDBS-TX100 dry into a uniform thin structures. In all of the above cases, SWNT-SDBS dispersion had $0.1 \mathrm{wt} \%$ SWNT, $1 \mathrm{wt} \%$ SDBS; 3 wt \% TX100 was added to it to obtain the SWNTSDBS-TX100 dispersion.

ticular, SDBS-based dispersions had 5-fold performance advantage over films obtained from other dispersions.

These significant differences in properties can be understood based on the work by Hecht et $a l .^{45}$ on the conductivity $\sigma_{\mathrm{dc}}$ of SWNT films. These films have the morphology of disordered two-dimensional networks of SWNT bundles; their electrical conductivity is expected to depend on the average bundle length $L_{a v}$ and diameter $D_{\mathrm{av},} \sigma_{\mathrm{dc}} \approx D_{\mathrm{av}}^{-2}, \sigma_{\mathrm{dc}} \approx L_{\mathrm{av}}^{1.46}$. Compared to SDS and Triton X-100, SDBS is a better dispersing agent, yielding dispersions with higher concentration of individual SWNTs at the same overall SWNT concentration. ${ }^{46}$ These differences in the SWNT microstructure (e.g., ratio of individual vs bundles, average bundle diameter) in various dispersions are the likely cause of the different electro-optical properties of the final SWNT films.

TABLE 1. Relative Performance of "Test" Films Made from Coating Fluids Incorporating Different Surfactants ${ }^{a}$

\begin{tabular}{lcc}
$\begin{array}{c}\text { coating fluid } \\
\text { composition }\end{array}$ & $\begin{array}{c}\mathrm{FOM}_{1} \text { of transparent } \\
\text { conductive film }(\boldsymbol{\Omega} / \mathbf{s q})\end{array}$ & $\begin{array}{c}\text { FOM }_{2} \text { of transparent } \\
\text { conductive film }\end{array}$ \\
\hline SWNT + F108 & $472 \pm 98$ & $2.56 \pm 0.78$ \\
SWNT + F98 & $472 \pm 108$ & $3.35 \pm 2.12$ \\
SWNT + SDS & $274 \pm 83$ & $0.92 \pm 0.20$ \\
SWNT + SDBS & $52 \pm 12$ & $0.20 \pm 0.03$
\end{tabular}

${ }^{a}$ All coating fluids had $0.3-0.4$ wt \% SWNT and 1 wt $\%$ surfactant. The FOM reported in each case is an average over $5-6$ films within the transparency range of $20-50 \%$.
On the basis of these observations, SDBS was selected as the surfactant of choice. Attaining higher transparency in films required coating fluids with lower concentration of SWNTs ( $\sim 0.1 \mathrm{wt} \%$ for our rod coating setup), which had lower viscosity, required longer drying time, and therefore were more prone to forming defective films. The wetting and rheological properties of the SWNT dispersions were modified in order to eliminate film defects and nonuniformities due to dewetting and film rupture. Redon et al..$^{47}$ have studied the dynamics of dewetting and breakage of thin liquid films by contact line recession and growth of dry regions. They show that liquid films thinner than their characteristic equilibrium thickness $h_{\mathrm{e}}=2(\sigma /$ $\rho g)^{1 / 2} \sin (\theta / 2)$, where $\theta$ is the contact angle of the liquid with the substrate, $h_{\mathrm{e}} \approx 400 \mu \mathrm{m}$ for surfactant-water based systems, are metastable and always evolve toward equilibrium by either dewetting and shrinkage or by breaking up in to beads and patches. They also show that the dewetting velocity is $V_{\text {dewet }}=(k \sigma / \mu) \theta^{3}$, where $k$ is a fluid property and can be taken as $10^{-3}$ for primarily water-based systems. ${ }^{47} \mathrm{To}$ avoid film rupture, the dewetting velocity must be low so that the liquid film can dry before dewetting causes any defects; equivalently, dewetting is avoided when the film drying time is much shorter than the dewetting time $t_{\text {dewet }}=L / V_{\text {dewet, }}$ where $L$ is the characteristic length scale of the film. Therefore, dewetting can be avoided by lowering surface tension and contact angle, by raising viscosity, or by appropriately reducing the drying time of coated film.

The viscosity ranged from $6 \mathrm{mPa}$ s (at low shear rates) to $\sim 3 \mathrm{mPa} \cdot \mathrm{s}$ (at high shear rates). The surface tension and contact angle with clean glass surface for the 0.1 wt \% SWNT-SDBS dispersion were measured using a goniometer and were found to be $\sim 32 \mathrm{mN} / \mathrm{m}$ and $12^{\circ}$, respectively, which are typical for aqueous SDBS solutions above the critical micellar concentration (CMC of SDBS in water $\approx 0.08 \mathrm{wt} \%$ ). ${ }^{48}$ As the surfactant concentration of $1 \mathrm{wt} \%$ is safely above the CMC (even after accounting for surfactant adsorbed on SWNT surface, $\sim 0.4 \mathrm{wt} \%$ in case of SDBS and $0.1 \mathrm{wt} \%$ SWNT), the surface tension and contact angle are essentially independent of surfactant concentration. Therefore, we attempted to slow down dewetting by enhancing the viscosity of the coating fluid, with the constraint that the fluid should still have sufficiently low viscosity 
TABLE 2. List of Physical Properties, Coating Related Numbers, and Time-Scales for the SWNT-SDBS Dispersion and Modified Values after the Addition of Triton X-100

\begin{tabular}{|c|c|c|c|c|c|c|c|c|c|}
\hline & $\begin{array}{l}\text { surface tension } \\
\qquad(\mathrm{mN} / \mathrm{m})\end{array}$ & $\begin{array}{l}\text { low-shear viscosity } \\
\left.\text { (at } \sim 0.1 \mathrm{~s}^{-1}\right)(\mathrm{mPa}-\mathrm{s})\end{array}$ & $\begin{array}{l}\text { high-shear viscosity } \\
\left.\text { (at } \sim 40 \mathrm{~s}^{-1}\right)(\mathrm{mPa}-\mathrm{s})\end{array}$ & $\mathrm{Ca}$ & Re & $\begin{array}{l}t_{\text {level }} \\
(s)\end{array}$ & $\begin{array}{l}t_{\mathrm{dry}} \\
(\mathrm{s})\end{array}$ & $\begin{array}{c}t_{\text {dewet }} \\
(s)\end{array}$ & $\begin{array}{c}V_{\text {dewet }} \\
(\mu \mathrm{m} / \mathrm{s})\end{array}$ \\
\hline SWNT-SDBS & 31.8 & 6 & 3 & $1.89 \times 10^{-3}$ & 2 & $2.29 \times 10^{-3}$ & 530 & 21 & 47.63 \\
\hline SWNT-SDBS - TX100 & 31.4 & 2000 & 12 & $7.6 \times 10^{-3}$ & 0.5 & $9.28 \times 10^{-3}$ & 530 & 7092 & 0.14 \\
\hline
\end{tabular}

${ }^{a}$ The SWNT-SDBS dispersion had 0.1 wt \% SWNT and 1 wt \% SDBS; 3 wt \% Triton X-100 was added to get the modified SWNT-SDBS-TX100 dispersion. Ca, Re, and $t_{\text {level }}$ were calculated using high shear viscosity, $V_{\text {dewet }}$ and $t_{\text {dewet }}$ were calculated using low shear viscosity. Length scale used for $t_{\text {level }}$ and $R e$ was $2 \pi I=2 \mathrm{~mm}$ (typical wavelength of irregularities left behind by coating rods) and a length scale ( $L$ ) of $1 \mathrm{~mm}\left(\sim 10 \%\right.$ of total width of the coated film) was used for estimating $t_{\text {dewet }}$. An average liquid film thickness of $100 \mu \mathrm{m}$ is assumed for all cases.

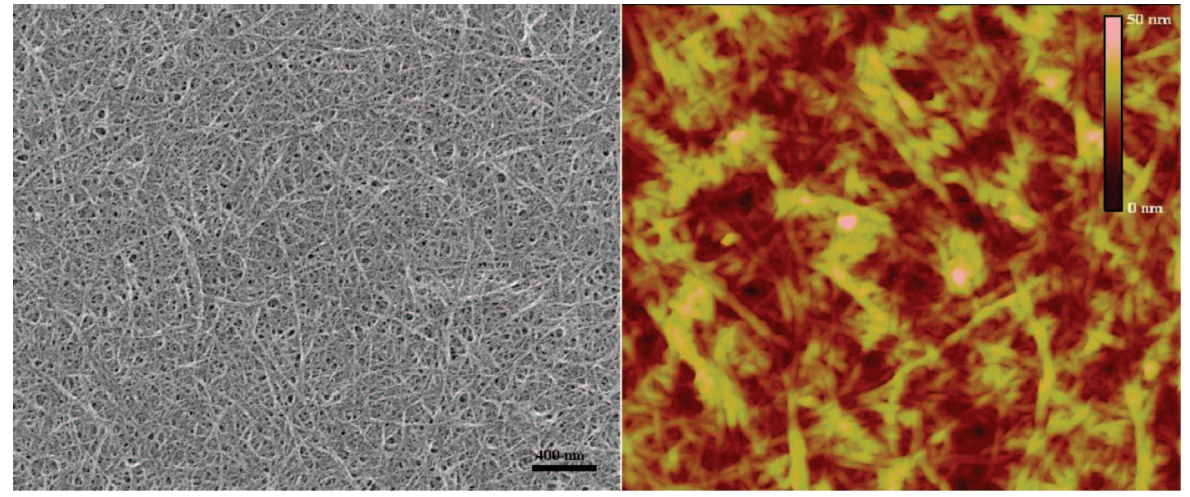

Figure 3. SEM and AFM images of SWNT thin film on glass fabricated using the draw-down rod coating technique using SWNT-SDBS - TX100 dispersion (SWNT $\approx 0.1 \mathrm{wt} \%$, SDBS $\approx 1 \mathrm{wt} \%$, TX100 $\approx 3 \mathrm{wt} \%$ ). Films coated with slightly different concentration of SWNT $(0.1-0.3 \mathrm{wt} \%)$ and TX100 (2-3\%) yielded similar microstructure. The scale bar in the SEM scan is 400 $\mathrm{nm}$ and the AFM image is a $2.5 \mu \mathrm{m} \times 2.5 \mu \mathrm{m}$ scan. The height scale bar in the AFM image is $50 \mathrm{~nm}$.

to allow deposition using coating rod and leveling of the surface waves immediately after deposition.

The options for enhancing the viscosity and controlling the fluid rheology were limited because the stability of the SWNT-SDSB dispersion is very sensitive to additives; moreover, an electrically insulating additive can be used only if it is easily washable from the solid film to avoid losing conductivity - this discourages the use of high molecular weight polymers. Triton X-100 (TX100), a nonionic surfactant, was found to have a tremendous effect on the viscosity of SWNT-SDBS dispersion. Figure 2 shows the steady shear viscosity of our coating fluid before and after the addition of TX100; adding TX100 to the SWNT-SDBS dispersion raised by about 3 orders of magnitude the zero-shear viscosity and only by 3 - to 5 -fold the high-shear viscosity.

The reason for the observed viscosity enhancement on the addition of TX100 is not entirely clear, as the exact nature of interaction between SDBS and TX100 in the presence of SWNTs has not been studied before. Plausible explanations can be proposed based on similar prior observations in closely related systems. The addition of a neutral or oppositely charged surfactant or salt to another pure surfactant solution raises significantly the viscosity of some systems. ${ }^{49,50}$ Adding a counterion or suitable cosurfactant results in dramatic changes in the surfactant micellar structure (e.g., from spherical to rodlike or wormlike), resulting in an equally dramatic increase in their shear viscosities. Previous studies $^{51,52}$ have shown that increasing the surfactant concentration in a SWNT-surfactant aqueous system leads to stickiness and depletion flocculation among SWNTs, which is also known to cause significant viscosity enhancements. ${ }^{53}$ Both these effects, which are due to the formation of weakly associated network structures, change the rheological behavior of the system, especially at low shear rates. At high shear rates, the effect on the rheological properties is less pronounced

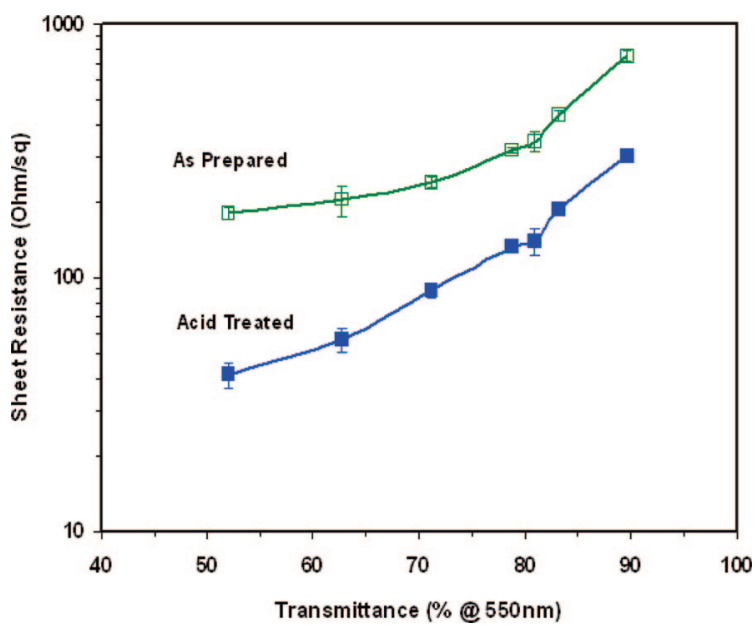

Figure 4. Sheet resistance versus transmittance for a series of rod-coated SWNT films with varying thicknesses, before and after oleum treatment. The films were coated using SWNT-SDBS-TX100 dispersion (SWNT $\approx 0.1 \mathrm{wt} \%$, SDBS $\approx$ $1 \mathrm{wt} \%$, TX100 $\approx 3 \mathrm{wt} \%$ ). The sheet resistance of all the films improved by over a factor of 3 upon acid treatment. The properties of the films were stable for over 8 weeks under ambient conditions.

VOL. $3 \cdot$ NO. $4 \cdot 835-843 \cdot 2009$ 


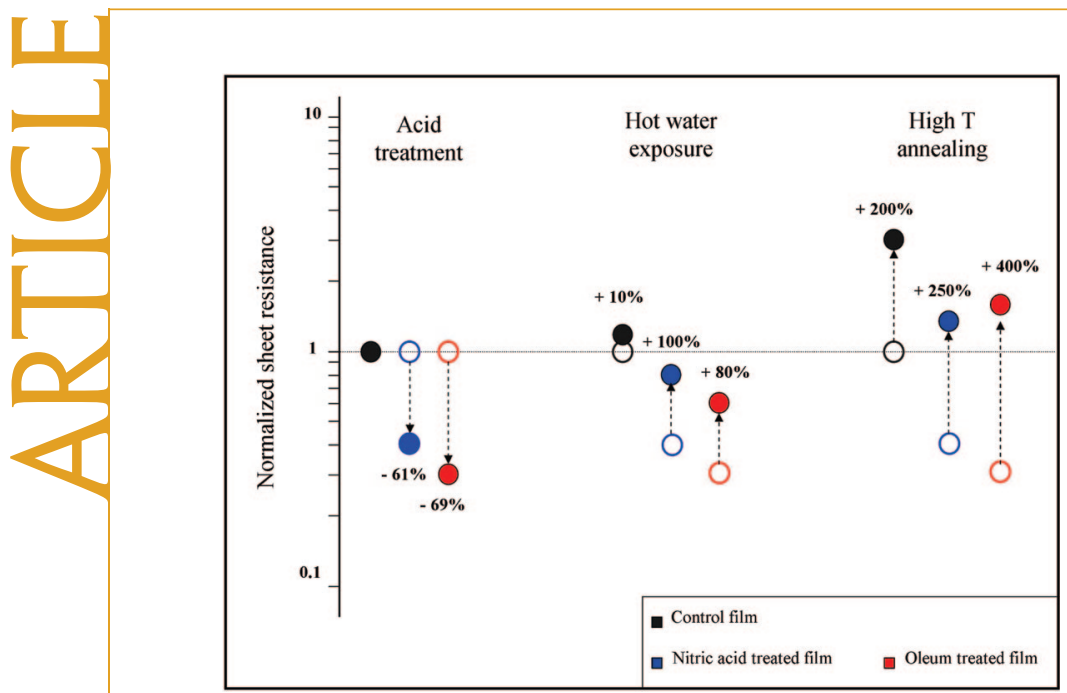

Figure 5. Effect of acid treatment and postprocessing (in $80^{\circ} \mathrm{C}$ hot water and $400{ }^{\circ} \mathrm{C} \mathrm{N}_{2}$ environment) on the electrical properties on SWNT films: (a) rod-coated, washed, but without any acid treatment, (b) rodcoated, washed, and dipped into $12 \mathrm{M}$ nitric acid, (c) rod-coated, washed, and dipped into oleum.

because shear stresses break flocs and micelle networks; similar to the trend observed in our SWNT-mixed surfactant system. Figure 2 shows the optical micrographs of SWNT-SDBS and SWNT-SDBS-TX100 dispersions.

Table 2 lists the capillary number, Reynolds number, dewetting velocity $\left(V_{\text {dewet }}\right)$ and the time scales for leveling, drying, and dewetting for both dispersions. The addition of TX100 slowed down the dewetting process (by significantly increasing the low-shear viscosity), while also keeping the capillary number sufficiently low $(<1)$. The leveling time $t_{\text {level }}$ is calculated using the high-shear viscosity, as it represents the state of the fluid close to the coating rod. The dewetting time (derived from $V_{\text {dewet }}$ ) is computed using the low-shear viscosity because the film is nearly stationary during dry-

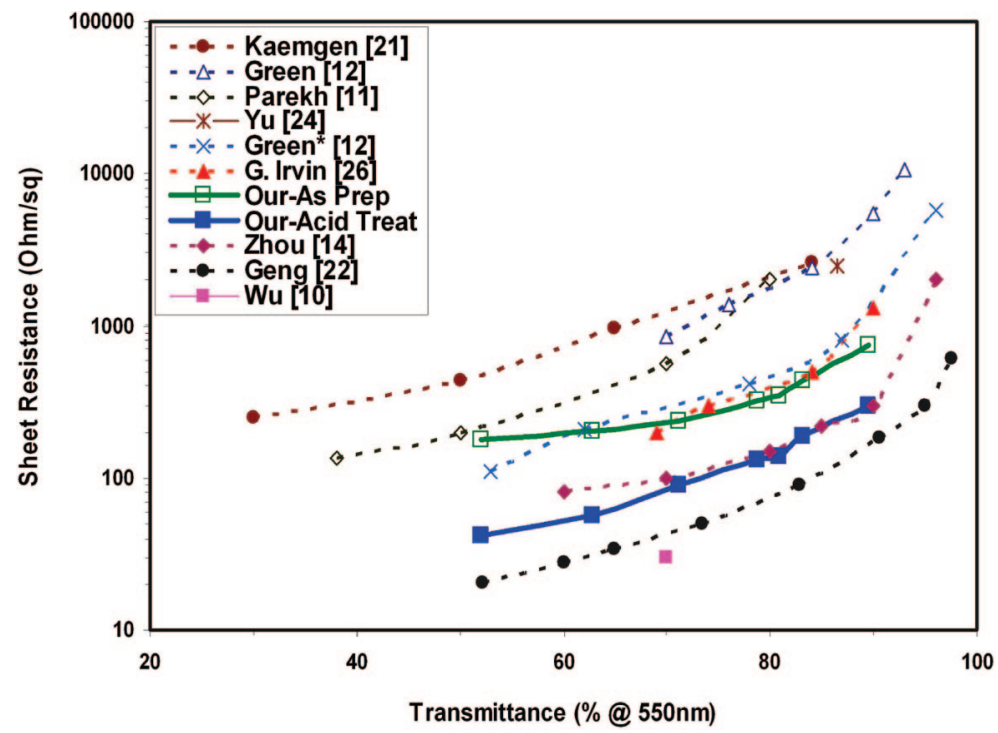

Figure 6. Comparison of the electro-optical performance of our acid-treated rodcoated SWNT films with other transparent conductive SWNT thin films published in the literature. ing. The time $t_{\mathrm{dry}}$ required to evaporate $80 \%$ of the solvent $\left(t_{\mathrm{dry}}=0.8 h_{\mathrm{o}} / J_{\mathrm{o}}\right)$ was estimated by using solvent evaporation current $J_{0}=1.5 \times 10^{-5} \mathrm{~cm} / \mathrm{s}$ obtained from a drop-drying experiment using SWNT-SDBS-TX100 dispersion (the same composition and ambient conditions used for rod coating); this evaporation current was then extrapolated to our $2.5 \mathrm{~cm} \times 5 \mathrm{~cm}$ area and 100 $\mu \mathrm{m}$ thick rod-coated thin film to obtain the reported $t_{\text {dry }}$. Adding Triton X-100 increased the dewetting time by two to 3 orders of magnitude, while the leveling time, critical for film smoothness, grew only by a factor of $\sim 4$ and remained well below the drying time.

Rod coating with the SWNT-SDBS-TX100 dispersion resulted in uniform, defect free thin film coatings. For electro-optical measurements, SWNT films of area 4 $\times 2 \mathrm{~cm}^{2}$ were coated on glass slides using the above dispersion. Hereafter the properties reported for each SWNT film represent the average of $4-5$ measurements performed at different locations of the film. Figure 2 shows a highly defective thin film coated on a microscope glass slide with the SWNT-SDBS dispersion and a uniform SWNT thin film coated with the SWNTSDBS-TX100 dispersion. Figure 3 shows the scanning electron microscope (SEM) image and atomic force microscope (AFM) image of the uniform film. Films as large as $20 \mathrm{~cm} \times 20 \mathrm{~cm}$ were fabricated on glass surfaces as well as commercially available PET substrates using the rod coater.

After washing the surfactants, the electrical conductivity of the SWNT film coatings was improved further by treatment with various acids. Although significant and stable improvement in the electrical properties of SWNT materials upon acid treatment has been reported before, ${ }^{22,54,55}$ the cause is still a matter of debate. Upon treatment with acids, Geng et al.22 observed a 5-fold increase of the electrical conductivity of SWNT thin films that had been made using a surfactant-based dispersion and had been washed to remove residual surfactant. They proposed that the acid removed residual surfactant molecules adsorbed on the surface of the nanotubes, leading to better contact between the nanotubes, densification of the films, and improvement in overall electrical conduction properties. However, work on macroscopic SWNT fibers ${ }^{54,55}$ spun from surfactant-free acid dispersions provides a different explanation. Ericson et al..$^{54}$ and Zhou et al..$^{55}$ showed by X-ray diffraction that strong acids like oleum penetrate SWNT bundles and networks forming a dense layer of intercalated anhydrous acid anions surrounding individual nanotubes. These physisorbed acid anions protonate and dope the nanotubes by forming charge-transfer complexes, improving their electrical conductivity. Acid doping is reversible and can be removed by heating the SWNTs in an inert or reducing environment. ${ }^{56,57}$ 
We acid treated our SWNT thin films by two different methods: wet treatment and vapor treatment. In wet treatment, the SWNT films were dipped in $12 \mathrm{M} \mathrm{ni-}$ tric acid or oleum $\left(\mathrm{H}_{2} \mathrm{SO}_{4}\right.$ : $20 \%$ excess $\left.\mathrm{SO}_{3}\right)$ for $30 \mathrm{~min}$ at room temperature, quenched for $30 \mathrm{~min}$ in diethyl ether or water and dried at $120^{\circ} \mathrm{C}$ under vacuum for $24 \mathrm{~h}$. For vapor treatment, the SWNT films were kept in a sealed, humidity-free environment saturated with acid vapors and maintained at high temperature $(\sim 70$ ${ }^{\circ} \mathrm{C}$ for nitric acid, $\sim 120{ }^{\circ} \mathrm{C}$ for oleum) for $30 \mathrm{~min}$, followed by 30 min of quenching in diethyl ether or water and drying at $120{ }^{\circ} \mathrm{C}$ under vacuum for $24 \mathrm{~h}$. Acid treatment by both methods improved significantly (at least 3 times) the electrical properties of the films while the transparency was unchanged. Figure 4 shows the sheet resistance versus transmittance curve for films with varying thicknesses, before and after the acid treatment (detailed visible-NIR transmission spectra of the SWNT films can be found in the Supporting Information). Sheet resistance as low as 80 and $140 \Omega /$ sq for corresponding transmittances of $70 \%$ and $80 \%$ were obtained after this process. The improvement obtained by acid treatment was highly reproducible and the sheet resistance of the films remained stable even after 8 weeks under ambient conditions.

We performed durability tests on our acid-treated as well as untreated SWNT films to study and compare the changes in their electrical conductivity on exposure to various harsh conditions. The tests were done using 3 film samples: (a) rod-coated and washed but without any acid treatment (control sample), (b) rod-coated, washed, and treated with $12 \mathrm{M}$ nitric acid and (c) rodcoated, washed, and treated with oleum. In one test, the films were immersed for $4 \mathrm{~h}$ in a water bath at $\sim 80$ ${ }^{\circ} \mathrm{C}$. In another test, the SWNT films were annealed for $4 \mathrm{~h}$ at $400{ }^{\circ} \mathrm{C}$ in an inert $\left(\mathrm{N}_{2}\right)$ atmosphere. The results of these tests are summarized in Figure 5. Both tests lowered the electrical conductivity of all three films. However, the loss of conductivity was much higher for acidtreated films than for the untreated film. Moreover, the extent of conductivity loss was different in the cases of nitric acid-treated film and oleum-treated film. These results show that the conductivity enhancement is due chiefly to the p-doping by acid anions intercalated and physisorbed in the SWNT network, although better removal of residual surfactant may also contribute to the effect.

Figure 6 compares the performance of our rollcoated SWNT films with other transparent conductive SWNT thin films published in the literature. Figure 7 shows the FOM plots for the same set of data using eqs 1 and 2. In Figure 7a the slopes of the curves are equal to $\alpha \rho\left(\mathrm{FOM}_{1}\right)$, whereas in Figure $7 \mathrm{~b}$ the slopes are $\left(Z_{0} / 2\right)\left(\sigma_{\mathrm{ac}} / \sigma_{\mathrm{dc}}\right)$ or $\left(Z_{0} / 2\right) \mathrm{FOM}_{2}$; in both cases, lower slope denotes more conductive and transparent material. As can be easily seen, both plots are essentially equivalent,
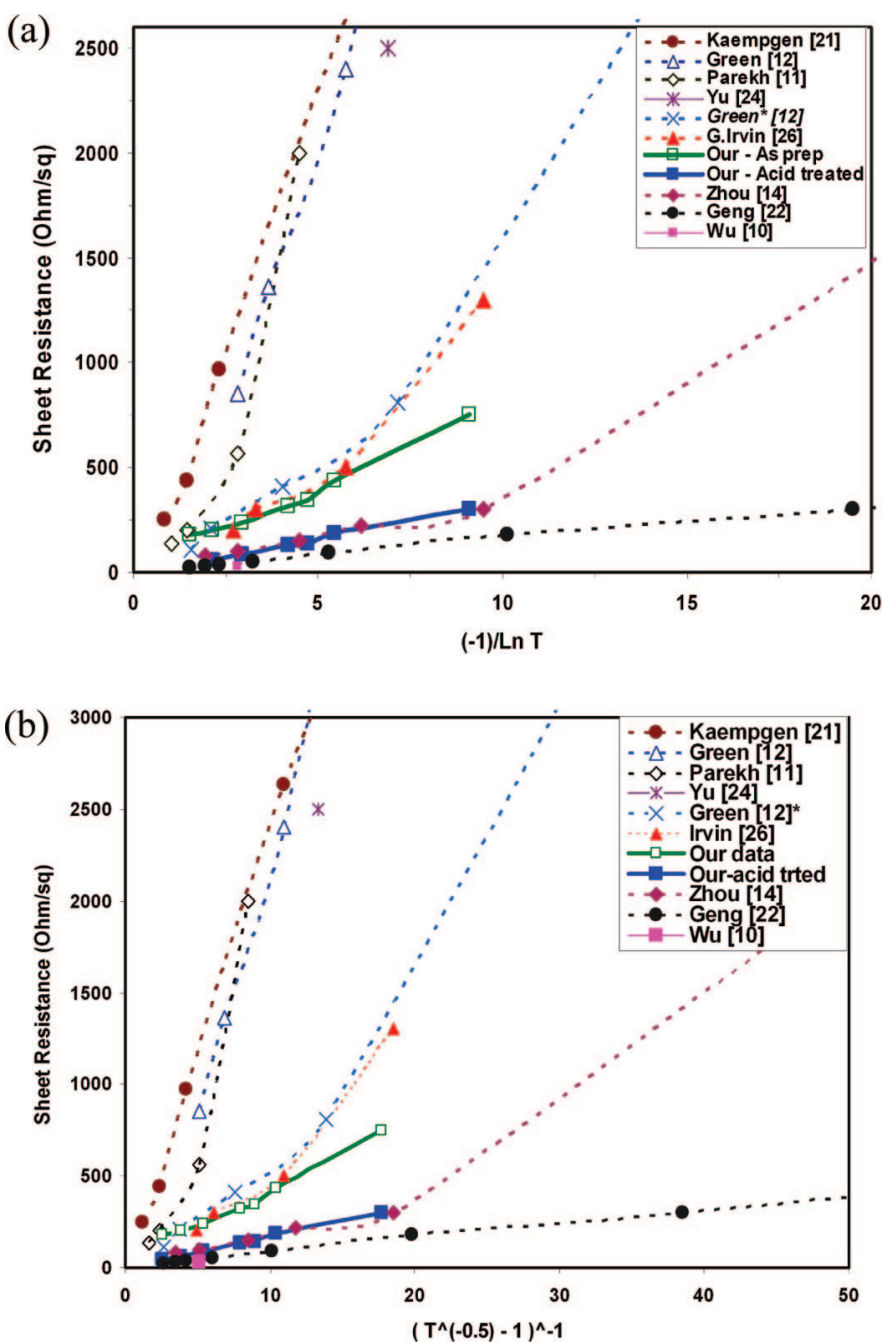

Figure 7. (a) FOM plot of our acid-treated rod-coated SWNT films and other transparent conductive SWNT thin films published in the literature, using eq 1. The slope of the lines, also referred to as $\mathrm{FOM}_{1}$, is the product of light absorption coefficient $(\alpha)$ and electrical resistivity $(\rho)$. (b) FOM plot of the same data set using eq 2 . Here, the slope of the lines is $\left(Z_{0} / 2\right) \cdot\left(\sigma_{a c} / \sigma_{d c}\right)$ or $\left(Z_{0} /\right.$ 2)FOM $\mathrm{F}_{2}$. Film series with lower slopes have better electro-optical performance (higher transparency for same resistance, and lower resistance for same transparency). Deviation from a straight line at high transparency (high values of $[-1 / \mathrm{ln} T]$ ) indicates the SWNT and SWNT bundles are losing percolation in the film. (Green [12]* represents the performance of metallic enriched SWNT films reported by Green et al. in ref 12).

showing similar trends (difference in slopes, deviations from straight line behavior, etc.) in a similar fashion.

The FOM plots show that films made from the same material (SWNTs) but by different methods can display substantially different intrinsic material properties (based on the slope of the curves). The electrical and optical properties of a SWNT thin film depend on the network microstructure, which is controlled by the fabrication process. The FOM plots also display the critical transparency beyond which the assumption of homogeneous film (linear plot) fails. At higher transparencies, films are thinner and the constituent SWNT networks approach the limit of percolation; once percolation is lost, the assumption of homogeneous material breaks down and the sheet resistance shoots up. Processes like 
rod coating maintain the linear relationship up to higher transparency because they yield better connected and packed SWNT networks; therefore, they are better suited for large-scale fabrication.

\section{CONCLUSION}

In summary, we present a highly scalable and continuous method for making uniform thin films of pristine SWNTs. A combination of surfactants was used to design a coating fluid with high concentration of SWNTs in water and optimal rheological and wetting behavior. The choice of surfactant had a strong effect on the rheological properties of the coating fluids as well as the electro-optical properties of the final SWNT films. Treatment with strong acids p-doped the SWNTs and improved the electrical conductivity of SWNT thin films by up to 5 -fold. The doping was stable at ambient conditions. The microstructure and electro-optical performance of the SWNT films was analyzed and compared with SWNT films fabricated by other methods using a FOM, showing that this method outperforms all other literature results except for recent air-spraying. The results presented here, both in terms of scientific understanding of how to control the fluid and process, and in terms of a scalable technique that can be extended to higher-throughput methods (slot, slide, and roll coating) paves the way to the deployment of transparent conductive SWNT films in large scale commercial applications.

Acknowledgment. The authors thank W. Suszynski, H. K. Schmidt, R. Hauge, G. Hirasaki, C. Miller, M. Majumdar, N. ParraVasquez, N. Alvarez, R. Duggal, R. Sharma, A. Mayeux, K. Fair, and C. Buxton for helpful discussions and valuable inputs. We also thank J. Tour group at Rice University for help with the electrical measurements. We gratefully acknowledge funding from the Air Force Office of Scientific Research under grant FA9550-06-10207, Air Force Research Laboratory under grant 07-S568-004201-C1, and U.S. Army Corps of Engineers Environmental Quality and Installation Program under grant W912HZ-08-C-0054. This article is dedicated to Skip Scriven.

Supporting Information Available: Materials and Methods. This material is available free of charge via the Internet at http:// pubs.acs.org.

\section{REFERENCES AND NOTES}

1. Tans, S. J.; Devoret, M. H.; Dai, H.; Thess, A.; Smalley, R. E.; Geerligs, L. J.; Dekker, C. Individual Single-Wall Carbon Nanotubes as Quantum Wires. Nature (London) 1997, 386, 474-477.

2. Liang, W. J.; Bockrath, M.; Bozovic, D.; Hafner, J. H.; Tinkham, M.; Park, H. Fabry-Perot Interference in a Nanotube Electron Waveguide. Nature (London) 2001, 411, 665-669.

3. Maeda, A.; Matsumoto, S.; Kishida, H.; Takenobu, T.; Iwasa, Y.; Shiraishi, M.; Ata, M.; Okamoto, H. Large Optical Nonlinearity of Semiconducting Single-Walled Carbon Nanotubes under Resonant Excitations. Phys. Rev. Lett. 2005, 94, 047404.

4. Tatsuura, S.; Furuki, M.; Sato, Y.; Iwasa, I.; Tian, M.; Mitsu, H. Semiconductor Carbon Nanotubes as Ultrafast Switching Materials for Optical Telecommunications. Adv. Mater. 2003, 15, 534 .
5. Yakobson, B. I.; Avouris, P. Mechanical Properties of Carbon Nanotubes. In Carbon Nanotubes; Springer-Verlag Berlin: Berlin, 2001; Vol. 80, pp 287-327.

6. Serp, P.; Corrias, M.; Kalck, P. Carbon Nanotubes and Nanofibers in Catalysis. Appl. Catal. A 2003, 253, 337-358.

7. Baughman, R. H.; Zakhidov, A. A.; de Heer, W. A. Carbon Nanotubes-The Route Toward Applications. Science 2002, 297, 787-792.

8. Chopra, K. L.; Major, S.; Pandya, D. K. Transparent Conductors-A Status Review. Thin Solid Films 1983, 102, $1-46$.

9. Granqvist, C. G.; Hultåker, A. Transparent and Conducting ITO Films: New Developments and Applications. Thin Solid Films 2002, 411, 1-5.

10. Wu, Z.; Chen, Z.; Du, X.; Logan, J. M.; Sippel, J.; Nikolou, M.; Kamaras, K.; Reynolds, J. R.; Tanner, D. B.; Hebard, A. F.; et al. Transparent, Conductive Carbon Nanotube Films. Science 2004, 305, 1273-1276.

11. Parekh, B. B.; Fanchini, G.; Eda, G.; Chhowalla, M. Improved Conductivity of Transparent Single-Wall Carbon Nanotube Thin Films via Stable Postdeposition Functionalization. Appl. Phys. Lett. 2007, 90, 121913.

12. Green, A. A.; Hersam, M. C. Colored Semitransparent Conductive Coatings Consisting of Monodisperse Metallic Single-Walled Carbon Nanotubes. Nano Lett. 2008, 8, 1417-1422.

13. Hu, L.; Hecht, D. S.; Gruner, G. Percolation in Transparent and Conducting Carbon Nanotube Networks. Nano Lett. 2004, 4, 2513-2517.

14. Zhou, Y.; Hu, L.; Grüner, G. A Method of Printing Carbon Nanotube Thin Films. Appl. Phys. Lett. 2006, 88, 123109.

15. Zhang, M.; Fang, S.; Zakhidov, A. A.; Lee, S. B.; Aliev, A. E.; Williams, C. D.; Atkinson, K. R.; Baughman, R. H. Strong, Transparent, Multifunctional, Carbon Nanotube Sheets. Science 2005, 309, 1215-1219.

16. Meitl, M. A.; Zhou, Y. X.; Gaur, A.; Jeon, S.; Usrey, M. L.; Strano, M. S.; Rogers, J. A. Solution Casting and Transfer Printing Single-Walled Carbon Nanotube Films. Nano Lett. 2004, 4, 1643-1649.

17. Sreekumar, T. V.; Liu, T.; Kumar, S.; Ericson, L. M.; Hauge, R. H.; Smalley, R. E. Single-Wall Carbon Nanotube Films. Chem. Mater. 2003, 15, 175-178.

18. Armitage, N. P.; Gabriel, J. C. P.; Gruner, G. QuasiLangmuir-Blodgett Thin Film Deposition of Carbon Nanotubes. J. Appl. Phys. 2004, 95, 3228-3230.

19. Spotnitz, M. E.; Ryan, D.; Stone, H. A. Dip Coating for the Alignment of Carbon Nanotubes on Curved Surfaces. J. Mater. Chem. 2004, 14, 1299-1302.

20. Ma, W.; Song, L.; Yang, R.; Zhang, T.; Zhao, Y.; Sun, L.; Ren, Y.; Liu, D.; Liu, L.; Shen, J. Directly Synthesized Strong, Highly Conducting, Transparent Single-Walled Carbon Nanotube Films. Nano Lett. 2007, 7, 2307-2311.

21. Kaempgen, M.; Duesberg, G. S.; Roth, S. Transparent Carbon Nanotube Coatings. Appl. Surf. Sci. 2005, 252, 425429.

22. Geng, H. Z.; Kim, K. K.; So, K. P.; Lee, Y. S.; Chang, Y.; Lee, Y. H. Effect of Acid Treatment on Carbon Nanotube-Based Flexible Transparent Conducting Films. J. Am. Chem. Soc. 2007, 129, 7758-7759.

23. Yu, X.; Rajamani, R.; Stelson, K. A.; Cui, T. Carbon Nanotube Based Transparent Conductive Thin Films. J. Nanosci. Nanotechnol. 2006, 6, 1939-1944.

24. Yu, X.; Rajamani, R.; Stelson, K. A.; Cui, T. Fabrication of Carbon Nanotube Based Transparent Conductive Thin Films Using Layer-By-Layer Technology. Surf. Coat. Technol. 2008, 202, 2002-2007.

25. Rowley, L. A.; Irvin, G. C.; Anderson, C. C.; D., M. Coating Compositions Containing Single Wall Carbon Nanotubes. U.S. Patent Appl. 20060188723, 2006.

26. Rowley, L. A.; Spath, T. M.; Irvin, G. C. Roll to Roll High Transparency, High Conductivity SWCNT Films Used in Flexible Electronics. Abstr. Am. Chem. Soc. 2006, 232, 130PRES.

27. Mayer, C. W., Coating-Machine. U.S. Patent 1043021, 1911. 
28. Cohen, E.; Gutoff, E. Modern Coating and Drying Technology. In Choosing the Coating Method; Cohen, E., Ed.; VCH Publishers: New York, 1992; pp 6-7.

29. Kistler, S. F.; Schweizer, P. M. Liquid Film Coating: Scientific Principles and Their Technological Implications; Chapman \& Hall: New York, 1997.

30. Keunings, R.; Bousfield, D. Analysis of Surface-Tension Driven Leveling in Viscoelastic Films. J. Non-Newtonian Fluid Mech. 1987; 22, 219-233.

31. Coyle, D. J.; Macosko, C. W.; Scriven, L. E. Reverse Roll Coating of Non-Newtonian Liquids. J. Rheol. 1990, 34, 615.

32. Pasquali, M.; Scriven, L. E. Free Surface Flows of Polymer Solutions with Models Based on the Conformation Tensor. J. Non-Newtonian Fluid Mech. 2002, 108, 363-409.

33. Hobbie, E. K.; Fry, D. J. Rheology of Concentrated Carbon Nanotube Suspensions. J. Chem. Phys. 2007, 126, 124907.

34. Parra-Vasquez, A. N. G.; Stepanek, I.; Davis, V. A.; Moore V. C.; Haroz, E. H.; Shaver, J.; Hauge, R. H.; Smalley, R. E.; Pasquali, M. Simple Length Determination of SingleWalled Carbon Nanotubes by Viscosity Measurements in Dilute Suspensions. Macromolecules 2007, 40, 4043-4047.

35. Rai, P. K.; Parra-Vasquez, A. N. G.; Chattopadhyay, J.; Pinnick, R. A.; Liang, F.; Sadana, A. K.; Hauge, R. H.; Billups, W. E.; Pasquali, M. Dispersions of Functionalized SingleWalled Carbon Nanotubes in Strong Acids: Solubility and Rheology. J. Nanosci. Nanotechnol. 2007, 7, 3378-3385.

36. Kinloch, I. A.; Roberts, S. A.; Windle, A. H. A Rheological Study of Concentrated Aqueous Nanotube Dispersions. Polymer 2002, 43, 7483-7491.

37. Moore, V. C.; Strano, M. S.; Haroz, E. H.; Hauge, R. H. Smalley, R. E.; Schmidt, J.; Talmon, Y. Individually Suspended Single-Walled Carbon Nanotubes in Various Surfactants. Nano Lett. 2003, 3, 1379-1382.

38. O'Connell, M. J.; Boul, P.; Ericson, L. M.; Huffman, C.; Wang, Y.; Haroz, E.; Kuper, C.; Tour, J.; Ausman, K. D.; Smalley, R. E. Reversible Water-Solubilization of Single-Walled Carbon Nanotubes by Polymer Wrapping. Chem. Phys. Lett. 2001, 342, 265-271.

39. Vigolo, B.; Penicaud, A.; Coulon, C.; Sauder, C.; Pailler, R.; Journet, C.; Bernier, P.; Poulin, P. Macroscopic Fibers and Ribbons of Oriented Carbon Nanotubes. Science $\mathbf{2 0 0 0}$ 290, 1331-1334.

40. Fraser, D. B.; Cook, H. D. Highly Conductive, Transparent Films of Sputtered InSnO. J. Electrochem. Soc. 1972, 119, 1368.

41. Haacke, G. New Figure of Merit for Transparent Conductors. J. Appl. Phys. 1976, 47, 4086.

42. Spath, T. M.; Irvin, G. C. J.; Majumdar, D.; Cok, R. S.; Anderson, C. C. Touchscreen with Conductive Layer Comprising Carbon Nanotubes. U.S. Patent Appl. 20060274048, 2006

43. Dressel, M.; Grüner, G. Electrodynamics of Solids. Cambridge University Press: New York, 2002.

44. Doherty, E.; De, S.; Lyons, P. E.; Shmelov, A.; Nirmalraj, P. N.; Scardaci, V.; Blau, W. J.; Boland, J. J.; Coleman, J. N. The Spatial Uniformity and Electromechanical Stability of Transparent, Conductive Films of Single Walled Nanotubes. Submitted for publication.

45. Hecht, D.; Hu, L.; Grüner, G. Conductivity Scaling with Bundle Length and Diameter in Single Walled Carbon Nanotube Networks. Appl. Phys. Lett. 2006, 89, 133112.

46. Islam, M. F.; Rojas, E.; Bergey, D. M.; Johnson, A. T.; Yodh, A. G. High Weight Fraction Surfactant Solubilization of Single-Wall Carbon Nanotubes in Water. Nano Lett. 2003, 3, 269-273.

47. Redon, C.; Brochard-Wyart, F.; Rondelez, F. Dynamics of Dewetting. Phys. Rev. Lett. 1991, 66, 715-718.

48. Mankowic, A. M. Dimensional Analysis Applied to Detergency. J. Am. Oil Chem. Soc. 1965, 42, 629-633.

49. Raghavan, S. R.; Fritz, G.; Kaler, E. W. Wormlike Micelles Formed by Synergistic Self-Assembly in Mixtures of Anionic and Cationic Surfactants. Langmuir 2002, 18 , 3797-3803.
50. Lin, Z.; Cai, J. J.; Scriven, L. E.; Davis, H. T. Spherical-toWormlike Micelle Transition in CTAB Solutions. J. Phys. Chem. 1994, 98, 5984-5993.

51. Wang, H.; Zhou, W.; Ho, D. L.; Winey, K. I.; Fischer, J. E.; Glinka, C. J.; Hobbie, E. K. Dispersing Single-Walled Carbon Nanotubes With Surfactants: A Small Angle Neutron Scattering Study. Nano Lett. 2004, 4, 1789-1793.

52. Vigolo, B.; Coulon, C.; Maugey, M.; Zakri, C.; Poulin, P. An Experimental Approach to the Percolation of Sticky Nanotubes. Science 2005, 309, 920-923.

53. Berli, C. L. A.; Quemada, D.; Parker, A. Modelling The Viscosity of Depletion Flocculated Emulsions. Colloids Surf., A 2002, 203, 11-20.

54. Ericson, L. M.; Fan, H.; Peng, H. Q.; Davis, V. A.; Zhou, W.; Sulpizio, J.; Wang, Y. H.; Booker, R.; Vavro, J.; Guthy, C.; et al. Macroscopic, Neat, Single-Walled Carbon Nanotube Fibers. Science 2004, 305, 1447-1450.

55. Zhou, W.; Fischer, J. E.; Heiney, P. A.; Fan, H.; Davis, V. A.; Pasquali, M.; Smalley, R. E. Single-Walled Carbon Nanotubes in Superacid: $\mathrm{X}$-ray and Calorimetric Evidence for Partly Ordered $\mathrm{H}_{2} \mathrm{SO}_{4}$. Phys. Rev. B 2005, 72, 45440.

56. Vavro, J.; Llaguno, M. C.; Fischer, J. E.; Ramesh, S.; Saini, R. K.; Ericson, L. M.; Davis, V. A.; Hauge, R. H.; Pasquali, M.; Smalley, R. E. Thermoelectric Power of p-Doped SingleWall Carbon Nanotubes and the Role of Phonon Drag. Phys. Rev. Lett. 2003, 90, 65503.

57. Zhou, W.; Vavro, J.; Guthy, C.; Winey, K. I.; Fischer, J. E.; Ericson, L. M.; Ramesh, S.; Saini, R.; Davis, V. A.; Kittrell, C.; et al. Single Wall Carbon Nanotube Fibers Extruded from Super-Acid Suspensions: Preferred Orientation, Electrical, and Thermal Transport. J. Appl. Phys. 2004, 95, 649-655. 